\title{
Nonlinear Shear Wave in a Non Newtonian Visco-elastic Medium
}

\author{
D. Banerjee M. S. Janaki and N. Chakrabarti \\ Saha Institute of Nuclear Physics, 1/AF Bidhannagar Calcutta - 700 064, India. \\ M. Chaudhuri \\ Max-Planck-Institut für extraterrestrische Physik, 85741 Garching, Germany
}

\begin{abstract}
An analysis of nonlinear transverse shear wave has been carried out on non-Newtonian viscoelastic liquid using generalized hydrodynamic $(\mathrm{GH})$ model. The nonlinear viscoelastic behavior is introduced through velocity shear dependence of viscosity coefficient by well known Carreau -Bird model. The dynamical feature of this shear wave leads to the celebrated Fermi-Pasta-Ulam (FPU) problem. Numerical solution has been obtained which shows that initial periodic solutions reoccur after passing through several patterns of periodic waves. A possible explanation for this periodic solution is given by constructing modified Korteweg de Vries $(\mathrm{mKdV})$ equation. This model has application from laboratory to astrophysical plasmas as well as biological systems.
\end{abstract}




\section{INTRODUCTION}

Viscoelastic properties of fluids in general and dusty plasmas in particular, have acquired high attraction recently due to the experimental characterization of non Newtonian nature and understanding of the associated physical processes. The interplay between shear flow and viscosity in a non Newtonian fluid, are purely nonlinear, therefore conventional linear theories are unfit to predict the behavior that what we observe in a medium. In response to external stresses viscoelastic media dissipate energy due to viscosity and store energy due to elastic nature. An important manifestation of such elastic property is reflected in the collective behavior with the prediction of a large amplitude transverse shear wave requesting some nonlinear physics.

Typically charged particle systems can be modeled as Yukawa systems that can have a liquid state $\underline{\underline{1}}-\underline{3}$ these include colloids, dense astrophysical plasmas $\frac{4}{4}$, strongly coupled dusty plasmas $\frac{5,6}{6}$ and magnetized plasma 7 . A Yukawa fluid possesses a memory dependent nonlocal viscoelastic coefficient enabling the propagation of a transverse shear wave that has been experimentally observed in a strongly coupled dusty plasma ${ }^{8}$. Another important characteristic exhibited by strongly coupled fluid is its non Newtonian behavior where the viscosity coefficient changes with velocity shear rate. This is also been observed in recent laboratory experiments ${ }^{9}-11$. Comparable examples of viscoelastic, non Newtonian behavior are also found in other branches of physics such as complex fluid ${ }^{12,13}$, polymeric liquids ${ }^{14}$, colloidal suspensions ${ }^{15}$, human blood 16 .

In this work, we construct a strongly nonlinear shear wave equation, for a non Newtonian viscoelastic fluid and find a possible solution of this nonlinear equation. The solution we found in a sense mimic the nonlinear solution of famous Fermi-Pasta-Ulam (FPU) problem ${ }^{17}$. Numerically integrated discrete equation is expanded in Taylor series in continuous variable and a dispersive effect is shown to exist and form a Modified Korteweg de Vries (mKdV) equation. The solution of $\mathrm{mKdV}$ equation helps us to explain the nonlinear periodic solutions.

\section{GENERALIZED HYDRODYNAMIC EQUATIONS WITH NON-NEWTONIAN VISCOSITY}

A strongly coupled dusty plasma whose constituents are electrons, ions and negatively fixed charged, massive dust grains are considered. The dust grains are strongly correlated to each other due to their larger electric charge and lower temperature, whereas, both electrons and ions are weakly coupled because of their smaller electric charges and higher temperatures. So the dynamics of shear waves in strongly coupled dusty plasma can be modeled by a memory dependent viscoelastic operator involving relaxation time $\tau_{m}$ that leads to generalized hydrodynamic momentum equation ${ }^{5,18}$ given by

$$
\left\{1+\tau_{m}\left(\frac{\partial}{\partial t}+\mathbf{v} \cdot \nabla\right)\right\}\left[\rho\left(\frac{\partial}{\partial t}+\mathbf{v} \cdot \nabla\right) \mathbf{v}-q n \mathbf{E}+\nabla p\right]=\frac{\partial \sigma_{i j}}{\partial x_{j}}
$$

where $\mathbf{v}, \rho, n, p, \mathbf{E}$ are respectively fluid velocity, mass density, number density, pressure, electric field and $\sigma_{i j}$ strain tensor of the medium. In a standard approach electrons and ions are light fluid and can be connected through electric field. However, for electrostatic shear wave, electric field will have no role to play hence explicit electron and ion dynamics are not taken into account. For an incompressible medium strain tensor is given by

$$
\sigma_{i j}=\eta(S)\left(\frac{\partial v_{i}}{\partial x_{j}}+\frac{\partial v_{j}}{\partial x_{i}}\right)
$$

For a Newtonian fluid $\eta$ is generally constant. However, for a non-Newtonian incompressible fluid, it has been shown that ${ }^{14}, \eta$ depends on the scalar invariants of strain tensor which can be written as

$$
I=\sum_{i} \sum_{j}\left(\frac{\partial v_{i}}{\partial x_{j}}+\frac{\partial v_{j}}{\partial x_{i}}\right)\left(\frac{\partial v_{i}}{\partial x_{j}}+\frac{\partial v_{j}}{\partial x_{i}}\right)
$$

and $S=\sqrt{I / 2}$. It has been shown that in the limit $\tau_{m} \partial / \partial t \gg 1$, viscoelastic fluid can support shear wave like transverse waves in elastic rods ${ }^{5}$. Since the motion is considered incompressible, $\rho$ remains constant in space and time and the velocity is the only dynamical variable. Our system of equations utilizes the geometry of shear waves, i.e. the $x$ and $t$ dependent velocity field in $y$-direction, the wave propagation is in $x$-direction. In such situation strain tensor $\sigma_{i j}$ and parameter $S$ are given by $\sigma_{y x}=\eta(S) \partial v_{y} / \partial x, S=\partial v_{y} / \partial x$. Using these, equation (1) is simplified as

$$
\frac{\partial^{2} v}{\partial t^{2}}=\frac{\partial}{\partial x}\left[\eta(S) \frac{\partial v}{\partial x}\right]
$$


To write the above equation we have used normalization as follows: $v \rightarrow v_{y} / c_{s h}, \eta \rightarrow \eta / \eta_{0}, x \rightarrow x / L, t \rightarrow t c_{s h} / L$ where, $\eta_{0}, L$, are some arbitrary viscosity and length, $c_{s h}=\sqrt{\eta_{0} / \rho \tau_{m}}$. To proceed further one needs to know the functional form of viscosity. In order to specify $\eta$ we have taken some input from recent experimental results 10 . In the experiment it was shown that charged fluids like dusty plasma have both shear thickening and thinning properties depending upon the parameter regime of shear rate. In order to include both properties in our solution we have taken well known Carreau-Bird viscosity mode ${ }^{14}$. There are other models but this model has an added advantage in a sense that one can easily recover the Newtonian viscosity. Mathematically this model is expressed as $\eta(S)=1+\alpha S^{2}$. The parameter $\alpha$ is used as a measure of non-Newtonian effect, and is assumed to be small in Carreau Bird model. Obviously for $\alpha$ negative(positive) the fluid behaves like a shear thinning(thickening) medium.

\section{NONLINEAR SHEAR WAVES, ENERGY SHARING, RECURRENCE}

Introduction of this model into equation (2), we obtain

$$
\frac{\partial^{2} v}{\partial t^{2}}=\frac{\partial^{2} v}{\partial x^{2}}+\alpha \frac{\partial}{\partial x}\left(\frac{\partial v}{\partial x}\right)^{3}
$$

As it is mentioned before, in absence of non-Newtonian effect i.e. $\alpha=0$ we get back the linear shear wave equation (normalized form) which resembles the wave equation in elastic media. The nonlinear wave equation (3) is characterized by a parameter $\alpha$ therefore the solution depends on a single independent external parameter.

Analytic solution is not straightforward therefore first we have studied this equation (3) numerically. Space is discretized with a minimum length $\Delta x$ and the equation is given by

$$
\frac{\partial^{2} v}{\partial t^{2}}=\frac{1}{(\Delta x)^{2}}\left(v_{j+1}-2 v_{j}+v_{j-1}\right)+\frac{\alpha}{(\Delta x)^{4}}\left[\left(v_{j+1}-v_{j}\right)^{3}+\left(v_{j-1}-v_{j}\right)^{3}\right]
$$

Next, we use mean value finite discretisation in time and solved this equation using a standard software $\frac{19,20}{2}$. In this numerical study, minimum space and time step introduce two parameters namely $\beta=(\Delta t / \Delta x)^{2}$ and $\gamma=\alpha \beta /(\Delta x)^{2}$. In a typical example we have used in the numerical investigation $\Delta t=0.03, \Delta x=0.04, \gamma \approx 351.5 \alpha$ with $\alpha=0.1$. In order to ensure the numerical stability, one must satisfy the Courant-Friedrichs-Lewy (CFL) condition. Initially $($ at $\mathrm{t}=0$ ), we perturb the system with a pure sine wave and keep observing its changes in time. After few time steps, wave is seen to change into a triangular shape. As time goes on, the amplitude of the wave form diminishes and the periodicity of the wave changes with time. Nonlinearity excites higher harmonics in the system and initial energy of fundamental mode is distributed through different harmonics(2). The interesting feature is that after a large number of time steps, all the higher modes starts to disappear and initial energy of the system accumulates in the fundamental mode(initial sinusoidal perturbation as shown in Fig (11). Therefore nonlinearity redistributes energy of the wave in different harmonics and they interact themselves and finally come back to its initial state. This feature of the solution reminds us the famous Fermi-Pasta -Ulam (FPU) problem in a completely different physical situation. Numerical analysis has also been carried out for shear thinning medium i.e when $\alpha$ is negative e.g, $\alpha=-0.3$. But, for this medium, energy sharing is negligible compared to that for shear thickening media and thus this case is not so interesting.

It is shown that the solutions of eq.(3) retrace the initial condition and maintain its periodicity inspite of the strong nonlinearity present in the equation. We search a possible explanation transforming the discrete equation to the continuum limit keeping higher space derivative in the Taylor series expansion of the velocity function. With an appropriate scaling $t \rightarrow(\Delta x) t$ and $\psi \rightarrow(\Delta x) \psi \sqrt{1 / 3 \alpha}$ in equation(44) and defining a continuum field variable $\psi(x, t)^{21}$, as

$$
\psi \equiv-\frac{v_{t}}{2(\Delta x)}+\frac{1}{2} \int_{0}^{v_{x}}\left(1 \pm(\Delta x)^{2} \zeta^{2}\right)^{1 / 2} d \zeta
$$

we have the equation

$$
\frac{\partial \psi}{\partial \tau} \pm 12 \psi^{2} \frac{\partial \psi}{\partial \xi}+\frac{\partial^{3} \psi}{\partial \xi^{3}}=0
$$

Note here that to write the above mKdV equation we have kept only lowest order terms in $\Delta x$ after Taylor expansion, with $\xi \equiv x-(\Delta x) t$ and $\tau \equiv(\Delta x)^{3} t / 24$. The mkdV equation given above has well known periodic solutions of the form $\sim c n^{2}$. This may be the case why we observe recurring solution in the numerical investigation of nonlinear shear wave equation. 

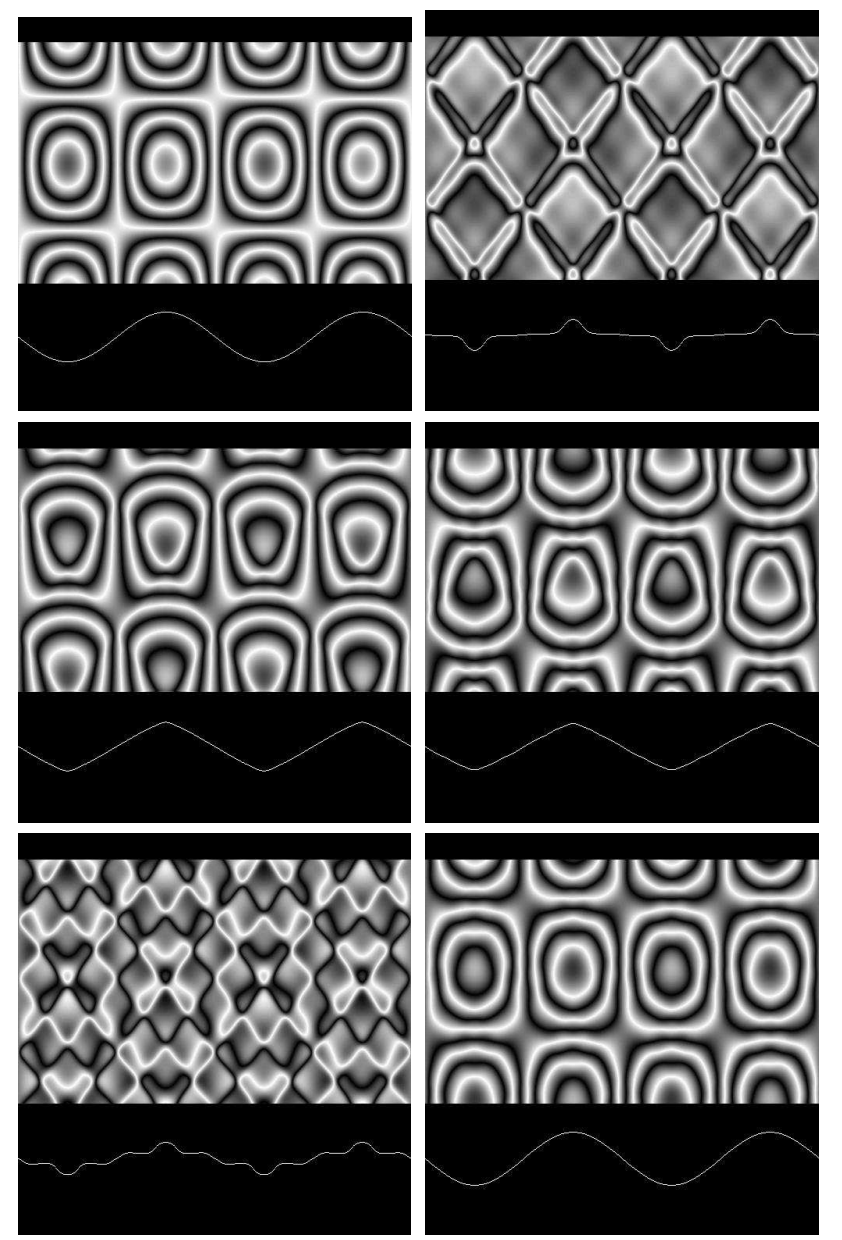

FIG. 1. Time evolution of the shear wave structure for $\alpha=0.1$ for different time(time increasing column wise) with initial and final state almost identical. $\mathrm{X}$-axis represents space and $\mathrm{y}$-axis represents time slots

\section{SUMMARY}

In conclusion, we emphasize that the analysis of a nonlinear shear wave has been done in a very simple macroscopic situation in which nonlinearity, viscosity and velocity shear are treated on equal footing, resulting in an interesting solution of the governing equations. The nonlinear shear wave equation is numerically solved by identifying it with the celebrated FPU problem with cubic nonlinearity. We could show that for the shear thickening situation nonlinear shear waves redistribute their energy and finally come back to the initial state while for a shear thinning medium energy distribution is negligibly small. Therefore the propagation of transverse shear waves is an important technique for characterization of strongly coupled Yukawa fluids. Our solution is well applicable to other branches of strongly coupled media such as neutral viscoelastic fluids ranging from polymer solutions, biological fluids like blood, pulmonary liquids, human soft tissues, to magma fluids. In such cases linear response is controlled by imposing small amplitude displacements. However, it is interesting and also desirable to extend such investigations to models with nonlinear constitutive equations leading to nonlinear physics. Such methods have the potential to characterize nonlinear viscoelastic properties at low stress levels that are typical in biological conditions. The important contribution of this work is to form and solve the evolution equation for nonlinear shear waves. This type of solution represents a new class of nonlinear solutions that may arise in a manifold of similar physical situations. The dependence of the time period of recurrence of periodic solutions on the nonlinearity parameter can enable the characterization of non-Newtonian properties possible. We believe that more investigations in this direction will enrich our knowledge 

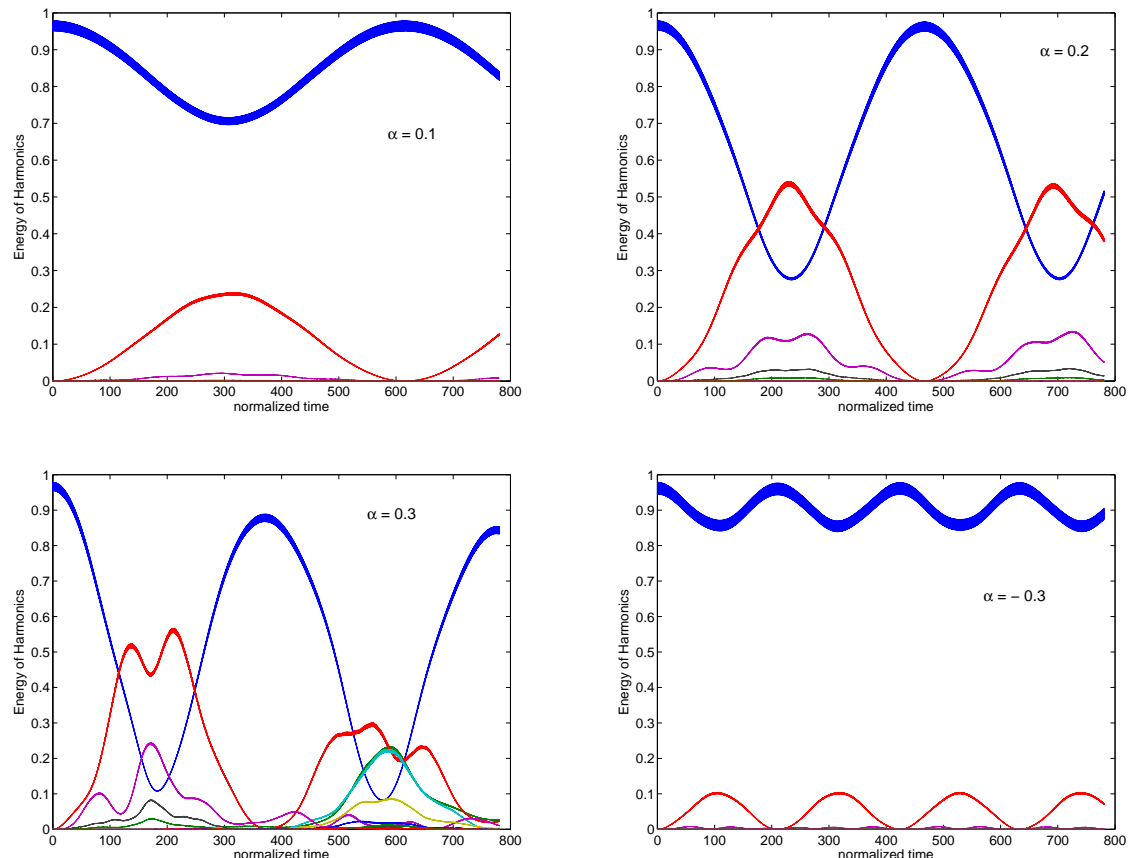

FIG. 2. Energy of different harmonics is plotted against normalized time for different values of nonlinearity $\alpha$. time is normalized by $c_{s h} / L$, where $L$ is the system length. Blue line shows energy of fundamental harmonic and other red, brown, yellow etc show for different higher harmonics. First three graphs show energy plots for three different positive values of $\alpha$. Hence, energy sharing to higher harmonics is greater for $\alpha=0.3$ than that of 0.1 and 0.2. Also recurrence is faster for larger values of $\alpha$. Fourth graph is for shear thinning medium.

on the physical understanding of strongly coupled media.

${ }^{1}$ P. K. Shukla and A. A. Mamun, Introduction to Dusty Plasma Physics (Institute of Physics Publication, Bristol, 2002)

${ }^{2}$ S. Ichimaru, Rev. Mod. Phys. 54, 1017 (1982)

3 P. K. Shukla, M. Torney, R. Bingham, and G. E. Morfill, Phys. Scripta. 67, 350 (2003)

${ }^{4}$ M. S. Janaki, N. Chakrabarti, and D. Banerjee, Phys. Plasmas 18, 012901 (2011)

${ }^{5}$ P. Kaw and A. Sen, Phys. Plasmas 5, $3552(1998)$

6 A. A. Mamun and P. K. Shukla, Euro. Phys. Letter 87, 55001 (2009)

7 D. Banerjee, M. S. janaki, and N. Chakrabarti, Phys. Plasmas 17, 113708 (2010)

8 J. Pramanik, G. Prasad, A. Sen, and P. Kaw, Phys. Rev. Lett. 88, 17500 (2002)

${ }^{9}$ V. Nosenko and J. Goree, Phys. Rev. Lett. 93, 155004 (2004)

10 A. V. Ivlev, V. Steinberg, R. Kompaneets, H. Höfner, I. Sidorenko, and G. E. Morfill, Phys. Rev. Lett. 98, 145003 (2007)

11 A. V. Gavrikov, D. N. Goranskaya, A. S. Ivanov, O. F. Petrov, R. A. Timirkhanov, N. A. Vorona, and V. E. Fortov, J. Plasma Physics 76, 579 (2010)

12 G. Sorasio, P. K. Shukla, and D. P. Resendes, New. J. Phys. 5, 81 (2003)

13 A. A. Mamun, P. K. Shukla, and T. Farid, Phys. Plasmas. 7, 2329 (2000)

${ }_{14}$ R. B. Bird, R. C. Armstrong, and O. Hassger, Dynamics of polymeric liquids Vol. 1 (Wiley, New York, 1977)

${ }^{15}$ E. E. B. White, M. Chellamuthu, and J. P. Rothstein, Rheol Acta 49, 119 (2010)

${ }^{16}$ G. Thurston, Biophys. J. 12, 1205 (1972)

17 E. Fermi, J. Pasta, and S. Ulam, Studies of Nonlinear Problem I (Los Alomas Report, LA-1940, 1955)

${ }^{18}$ Y. Frenkel, Kinetic Theory of Liquids (Clarendon, Oxford, 1946)

19 R. Rucker et. al., http://www.rudyrucker.com/capow software, downloaded (2007)

20 T. Dauxois, M. Peyrard, and S. Ruffo, European. J. Phys. 26, S3 (2005)

${ }^{21}$ C. F. Driscoll and T. M. O'Neil, Phys. Rev. Lett. 37, 89 (1976) 